\title{
Costos médicos directos de enfermedades neumocócicas invasoras y neumonías con diagnóstico radiológico en niños chilenos
}

\author{
Rosanna Lagos, ${ }^{1,2}$ Alma Muñoz, ${ }^{1}$ Aníbal Espinoza, ${ }^{3}$ Ángela Dowes, ${ }^{4}$ \\ Ricardo Ruttimann, ${ }^{5}$ Rómulo Colindres ${ }^{5}$ y Myron M. Levine ${ }^{4}$
}

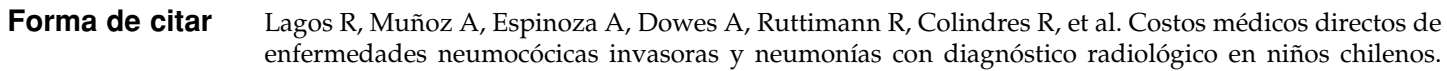
Rev Panam Salud Publica. 2009;26(2):101-11.

RESUMEN Objetivos. Determinar los costos médicos directos relacionados con la atención sanitaria de los casos de enfermedades neumocócicas invasoras (ENI) y neumonías adquiridas en la comunidad confirmadas mediante radiología (NAC-Rx) en niños chilenos.

Métodos. Estudio de seguimiento prospectivo de las prestaciones de salud entregadas a 594 niños de 0 a 35 meses con ENI y 1489 niños de 1 a 35 meses con NAC-Rx, diagnosticados y tratados en establecimientos de la red pública de salud de la Región Metropolitana de Chile. Las prestaciones se valoraron según las tarifas del Fondo Nacional de Salud (FONASA) y los precios de dos clínicas privadas. Se estimó la incidencia nacional anual de ENI y NAC-Rx para calcular la carga económica total nacional de la población afiliada al seguro de salud estatal.

Resultados. Los costos promedio de los casos que requirieron hospitalización fueron US\$ 1 056,20 para las ENI y US\$ 594,80 para las NAC-Rx, mientras que para los casos tratados en forma ambulatoria fueron US\$ 77,70 y US\$ 65,20, respectivamente. Los precios por los mismos servicios de internación fueron US\$ 484,10 y US\$2 962,70 en una de las clínicas privadas y US\$ 9 967,50 y US\$ 6 578,40 en la otra. El costo anual nacional estimado de la atención de los niños menores de 5 años según las tarifas de FONASA fue de US\$ 789045 para las ENI y US\$13 823289 para las NAC-Rx.

Conclusiones. La alta demanda asistencial y económica por NAC-Rx en niños de 0 a 3 años es una razón de salud pública tremendamente poderosa que apoya el uso sistemático de la vacunación antineumocócica en niños chilenos.

Palabras clave Costos directos de servicios; costos de la atención en salud; infecciones neumocócicas; vacunas neumocócicas; salud del niño; Chile.

1 Centro para Vacunas en Desarrollo-Chile, Santiago, Chile. La correspondencia se debe dirigir a Rosanna Lagos, Centro para Vacunas en Desarrollo-Chile, Hospital de Niños Roberto del Río, Avenida Zañartu No. 1085 ( $4^{\circ}$ Piso), Santiago, Chile. Correo electrónico: rosanna.lagos@adsl.tie.cl

2 Hospital de Niños Roberto del Río, Santiago, Chile.

3 Unidad de Imagenología, Clínica Alemana, Santiago, Chile.

4 Escuela de Medicina, Universidad de Maryland, Baltimore, Maryland, Estados Unidos de América.

5 GlaxoSmithKline-Biologicals, Río de Janeiro, Brasil.
Con la aparición de nuevas vacunas múltiples y el considerable aumento de sus precios, los argumentos de costos y beneficios han ido ganando hegemonía en las recomendaciones y las estrategias de uso de los nuevos productos en los programas globales y regionales de vacunación (1-4). Aunque existe un amplio consenso en que los análisis económicos aportan racionalidad y transparencia a la tarea de priorizar políticas de salud $(5,6)$, sus propios impulsores reconocen que la aplicación de estas herramientas en el ámbito de las decisiones nacionales sigue siendo insuficiente $(5,7)$. Una de las causas de esta contradicción es que la validez de los estudios económicos queda delimitada a su propio contexto, 
es decir, solo brindan orientaciones útiles dentro del escenario acotado por los parámetros específicos de cada análisis. Algunos parámetros críticos (como la carga de enfermedad, los costos de los tratamientos y de implementación, la cobertura de vacunación y la eficacia esperada) son especialmente variables en el campo de las enfermedades prevenibles mediante la adecuada vacunación. Por ello, los estudios económicos de vacunas suelen ser aún más restrictivos que los de otras intervenciones cuando se trata de extrapolar resultados de una comunidad a otra (8-10).

Las vacunas conjugadas contra Streptococcus pneumoniae han demostrado tener un enorme potencial para prevenir muertes y reducir la morbilidad infantil por infecciones invasoras (11-13), neumonías $(14,15)$ y otitis media $(16,17)$. Sin embargo, estas vacunas plantean a la vez un desafío presupuestario sin precedentes, especialmente para las agencias donantes y para los países de medianos ingresos que deben financiar sus programas de inmunización con fondos locales. Comprensiblemente, los análisis económicos de estas vacunas son materia de alto interés en la actualidad. La Alianza Global para Vacunas e Inmunizaciones (GAVI) comisionó un estudio para examinar la relación costo-efectividad del uso de vacunas conjugadas antineumocócicas en los 72 países que pueden optar por fondos de esta alianza (18). También el Instituto de Vacunas Albert Sabin, la Organización Panamericana de la Salud (OPS), los Centros para el Control y la Prevención de Enfermedades (CDC) de los Estados Unidos de América y el Plan para el Desarrollo y la Implementación Acelerada de las Vacunas Antineumocócicas (Pneumo-Adip) emprendieron recientemente un análisis similar, basado en estimaciones promedio de costos y carga de enfermedad neumocócica en países de América Latina y el Caribe (19). Tomando en cuenta la diversidad de las realidades epidemiológicas, económicas y de sistemas de salud que existen en la Región, la Unidad de Inmunizaciones de la OPS está liderando una vigorosa campaña para promover la realización de estudios locales que permitan reunir información económica científicamente validada para respaldar las decisiones de usar esta $u$ otras vacunas en los países de la Región de las Américas (20).

Mediante la combinación de los datos de carga de enfermedad recopilados en estudios epidemiológicos y las evidencias de eficacia y efectividad, ya sean nacionales o de otros países, se pueden construir modelos de análisis económico razonablemente ajustados a la realidad nacional para orientar decisiones sobre la incorporación de la vacuna antineumocócica a los programas de vacunación infantil.

El objetivo de este estudio fue determinar los costos médicos directos relacionados con la atención sanitaria de los casos de enfermedades neumocócicas invasoras (ENI) y neumonías adquiridas en la comunidad confirmadas mediante radiología (NAC-Rx) en niños chilenos.

\section{MATERIALES Y MÉTODOS}

Se realizó un estudio de seguimiento prospectivo de las prestaciones de salud entregadas a lactantes y niños de 0 a 35 meses con ENI y de 1 a 35 meses con NAC-Rx, diagnosticados y tratados en establecimientos de la red pública de salud de la Región Metropolitana (RM) de Chile, donde se ubica Santiago, la capital. La población de la RM es algo mayor de 6 millones de habitantes y representa casi $40 \%$ de la población del país. Entre $70 \%$ y $75 \%$ de los habitantes recibe atención de salud en el sistema estatal; el resto son afiliados a aseguradoras privadas (15-18\%), beneficiarios de sistemas cerrados pertenecientes a las Fuerzas Armadas y otras instituciones (6-7\%) y personas sin seguro médico (menos de $5 \%$ ). Más de $80 \%$ de las hospitalizaciones en niños residentes en la RM se realizan en 11 hospitales estatales dependientes del Ministerio de Salud, que en 2004 informaron 108353 egresos, 819153 consultas de urgencia y 475250 consultas de especialidades pediátricas (21).

En la RM, el servicio de atención primaria de salud opera mediante 140 consultorios gestionados en forma descentralizada por los 52 municipios. En cada municipio hay al menos un consultorio con capacidad para atender consultas médicas no programadas (servicios de atención primaria de urgencia) con horarios especiales, ya sean de 24 horas diarias los siete días de la semana, después del horario diurno regular (de las 17.00 a las 8.00 horas) o en días feriados. La oferta de estos servicios - que buscan reducir la carga de consultas de las unidades de emergencias de los hospitalesvaría en función de la demanda y tiene picos máximos durante las temporadas de infecciones respiratorias. El número anual de consultas pediátricas en estos servicios de atención primaria de urgencia ubicados en la RM es similar al de las unidades de urgencia de los hospitales.

\section{Casos de estudio}

Los casos de ENI se detectaron mediante la vigilancia iniciada en 1994 según una metodología ya publicada (22-25). Brevemente, enfermeras del Centro para Vacunas en Desarrollo de Chile (CVD-Chile) acudieron regularmente a los laboratorios de bacteriología de los establecimientos estatales de la RM para detectar aislamientos de S. pneumoniae en las muestras de sangre $\mathrm{u}$ otros fluidos corporales normalmente estériles procedentes de niños y posteriormente completaron un formulario estándar con los datos clínico-demográficos extraídos de la historia clínica de cada paciente. Los casos se agruparon en cinco categorías diagnósticas predefinidas: neumonía, neumonía con derrame o empiema pleural, meningitis, bacteriemia o sepsis sin foco, y otras formas clínicas de ENI con focalizaciones menos frecuentes, como peritonitis, infecciones osteoarticulares, infección de partes blandas, etc. Para el presente estudio se utilizaron los datos de los niños de 0 a 35 meses de edad con ENI diagnosticada entre el 1 de octubre de 2003 y el 30 de septiembre de 2006.

Los casos de NAC-Rx se tomaron de un estudio de carga de enfermedad realizado en seis municipios urbanos ubicados en la zona norte de la RM (Huechuraba, Conchalí, Independencia, Recoleta, Quilicura y Maipú) entre el 1 de diciembre de 2003 y el 31 de noviembre de 2005 (26). Los participantes eran niños de 1 a 35 meses de edad residentes en esos municipios, atendidos en los consultorios, los servicios de atención primaria de urgencia o en la unidad de urgencia del hospital pediátrico de la zona norte de la $\mathrm{RM}$, a quienes se les practicó una radiografía de tórax durante el curso de una infección respiratoria aguda o de un síndrome febril. Se excluyeron los casos con afecciones cardíacas, respiratorias o neurológicas crónicas graves. Las radiografías se digitalizaron en un aparato Vidar Sierra-PlusVXR (Vidar Systems Corporation, Herndon, VA, USA) y las imágenes digitales se interpretaron conforme al procedimiento estandarizado descrito por el Grupo de Trabajo de Neumonías de la Organización Mundial de la Salud 
(27). Los casos confirmados se agruparon en dos categorías radiológicas: neumonías consolidantes y neumonías no consolidantes. No se consideraron neumonías los casos que no presentaban infiltrados radiológicos consistentes con las definiciones preestablecidas.

La Unidad de Bioética del Ministerio de Salud revisó y aprobó los protocolos y los documentos de consentimiento de los estudios de ENI y NAC-Rx. El personal a cargo de la captación y el seguimiento de los participantes no intervino en las indicaciones de los estudios bacteriológicos y radiológicos, como tampoco en las decisiones de atención médica previas o posteriores a la inclusión de los casos.

\section{Datos para el análisis de costos}

Para el estudio de costos de las ENI se contactó a los padres de los casos incluidos, en un plazo no mayor de una semana desde la identificación bacteriológica de S. pneumoniae y se les solicitó el consentimiento informado para realizar el seguimiento detallado del tipo y el número de prestaciones de salud brindadas al paciente, desde el inicio de los síntomas hasta el alta médica o durante un máximo de seis meses posteriores al diagnóstico bacteriológico. Las prestaciones de interés para el estudio fueron la estadía hospitalaria, la atención por profesionales de la salud, los exámenes de laboratorio e imagenología, los procedimientos diagnósticos o terapéuticos y los tratamientos antimicrobianos administrados durante 3 días o más. La información se recogió en forma prospectiva durante la evolución clínica de los casos, mediante revisiones sucesivas de las fichas clínicas y contactos regulares con los padres. Los datos se registraron manualmente en un formulario individual creado para ello y posteriormente se introdujeron en una base de datos en Microsoft Access. Los casos en que no se obtuvo consentimiento paterno o materno se consideraron en las estimaciones de la incidencia de ENI, pero no aportaron datos para el estudio de costos.

Los procedimientos y los datos para el estudio de costos de los casos de NAC-Rx fueron similares a los descritos para los de ENI. Debido a que la mayoría de estos pacientes recibió la totalidad o parte de su tratamiento en forma ambulatoria, se solicitó a los padres que aceptaron participar que llevaran un registro de las prestaciones de interés en un formulario diseñado para este fin. El personal a cargo del seguimiento se mantuvo en contacto telefónico con los padres durante la evolución de la enfermedad y realizó visitas domiciliarias para retirar los formularios una vez que los participantes recibieron el alta médica. Las anotaciones de los padres se verificaron y complementaron con los datos registrados en la ficha clínica de cada participante.

\section{Valoración de las prestaciones}

Los servicios prestados se valoraron de acuerdo con las tarifas del Fondo Nacional de Salud (FONASA) correspondientes a 2006 y los precios de dos clínicas privadas, una utilizada por personas de nivel socioeconómico medio y otra que atiende una población de ingresos altos, ambas cubiertas por seguros de salud privados medios y altos, respectivamente. FONASA es el organismo público encargado de recaudar y administrar el presupuesto del sistema de seguro de salud estatal chileno y de facilitar los servicios que requieren sus afiliados mediante el pago o el copago a los establecimientos proveedores. Para una misma prestación, la tarifa de FONASA varía según el nivel de complejidad del establecimiento proveedor. En este estudio se utilizaron las tarifas máximas fijadas por FONASA para los establecimientos estatales.

Los costos de los tratamientos antimicrobianos según FONASA se calcularon a partir del catálogo de precios de la Central Nacional de Abastecimientos correspondiente a 2006 (28); esta entidad estatal vende medicamentos e insumos clínicos a los establecimientos asistenciales de la red pública de salud. Las dos clínicas privadas informaron los costos de los tratamientos antimicrobianos administrados a los pacientes hospitalizados. Como estas instituciones no entregan medicamentos a pacientes ambulatorios, los costos de estos tratamientos fueron calculados a partir de la lista de precios de una de las principales cadenas de farmacias del país.

Todos los costos se expresaron en dólares estadounidenses (US\$), según la tasa de cambio promedio del peso chilenos (\$) en 2006 publicada por el Banco Central de Chile (US\$1,00 = \$530,30) (29).

\section{Análisis de los datos}

Los datos se analizaron mediante indicadores descriptivos. Para cada categoría clínica de ENI y radiológica de NAC-Rx se contabilizó el total de prestaciones de cada tipo y el costo promedio por episodio según los tres regímenes tarifarios analizados. Los pacientes que permanecieron en un servicio hospitalario por 24 horas o más durante el curso de la enfermedad se contabilizaron como casos hospitalizados. Los costos de los casos hospitalizados y ambulatorios pertenecientes a una misma categoría diagnóstica se calcularon por separado.

La carga económica anual secundaria al cuidado médico (estadía hospitalaria, atención por profesionales, exámenes de laboratorio e imagenología, procedimientos diagnósticos o terapéuticos y tratamientos antimicrobianos) de los lactantes y los niños con ENI y NAC-Rx se calculó desde la perspectiva de FONASA - por extrapolación a partir de las cifras de incidencia estimadas para la RM durante el período de los respectivos estudios de costos-y el total nacional de la población de 0 a 59 meses (ENI) y de 1 a 35 meses (NAC-Rx) atendidos en el sistema de salud estatal. Aunque el estudio de costos de ENI no abarcó a los pacientes de 36 a 59 meses de edad, se consideró que los pacientes de este grupo de edad requirieron el mismo tipo y número de prestaciones que los niños menores de 3 años.

La incidencia anual de niños de 0 a 35 meses de edad con ENI atendidos en FONASA se ajustó a partir de los resultados de dos estudios $(30,31)$. En el primero se documentó que la proporción de niños menores de 3 años con fiebre alta (temperatura rectal de $40{ }^{\circ} \mathrm{C}$ o mayor) que acuden a los servicios de atención primaria de urgencia es similar a la observada en las unidades de urgencia de los hospitales y que la frecuencia de bacteriemia neumocócica en pacientes con esas características es similar en ambos tipos de establecimiento (30). Durante el período de recolección de datos para el estudio de costos de los casos de ENI, $2,9 \%$ de los servicios prestados en las unidades de urgencia correspondió a niños menores de 3 años con fiebre alta y 1,1\% de los hemocultivos practicados a estos pacientes resultaron positivos a $S$. pneumoniae. En el segundo estudio se encontró que 12,3\% de los menores de 3 años atendidos en las unidades de urgencia tenía fiebre moderada (temperatura rectal entre $39^{\circ} \mathrm{C}$ y $40{ }^{\circ} \mathrm{C}$ ) y en $0,7 \%$ de los hemocultivos se aisló $S$. pneumoniae (31). Estos porcentajes de consultas de pacientes con fiebre alta $(2,9 \%)$ y moderada $(12,3 \%)$ y de aislamiento de $S$. pneumoniae $(1,1 \%$ y $0,7 \%$, respectivamente) se aplica- 
ron al total de los servicios prestados a los niños de 0 a 35 meses registrados en las unidades de urgencia y los servicios de atención primaria de urgencia. La suma de estos casos (teóricos) y de los efectivamente detectados en los laboratorios de bacteriología se empleó como numerador para calcular la incidencia corregida de ENI en niños de 0 a 35 meses servidos por FONASA durante el período de octubre de 2003 a septiembre de 2006. No se realizaron correcciones a la incidencia de ENI en la población de 36 a 59 meses, porque no existe información acerca del número de consultas por síndrome febril o sobre la frecuencia de bacteriemia neumocócica en pacientes ambulatorios de este grupo de edad.

\section{RESULTADOS}

\section{Características clínico-demográficas de la población de estudio}

Entre el 1 de octubre de 2003 y el 30 de septiembre de 2006, los laboratorios de bacteriología de los 11 establecimientos estatales que atienden niños en la RM documentaron 640 casos de ENI en menores de 3 años; de ellos 338 presentaban signos o síntomas que motivaron su hospitalización inmediata y 302 se identificaron por un hemocultivo practicado durante una visita ambulatoria a una unidad de urgencia. De estos últimos, 55 quedaron internados en el momento de ser reevaluados con el resultado del hemocultivo: 1 había desarrollado meningitis, 1 presentaba infección de partes blandas, 33 tenían neumonía y 20 ingresaron para tratamiento antimicrobiano intravenoso, aunque no presentaban signos de focalización. Los 247 pacientes restantes también tuvieron una reevaluación médica tras conocerse el resultado del hemocultivo, pero continuaron con tratamiento ambulatorio.

En conjunto, $393(61,4 \%)$ de los 640 niños con ENI detectados a lo largo de los tres años del estudio de costos requirieron cuidados hospitalarios durante al menos 24 horas y 15 fallecieron, para una letalidad total de 2,3\%. La mayor letalidad específica se observó en los pacientes con meningitis (10 de $47 ; 21,3 \%$ ), seguida de la neumonía (4 de $318 ; 1,3 \%$ ); ocurrió una sola muerte entre los 56 pacientes con empiema (1,8\%).

En 594 (92,8\%) de los 640 casos elegibles se obtuvo autorización paterna o materna para investigar las prestaciones de salud recibidas. Comprensiblemente, la tasa de reclutamiento fue sustancialmente inferior entre los pacientes que fallecieron (4 casos de los 15 fallecidos aportaron datos; 26,7\%).

De los 1553 casos de NAC-Rx estudiados, 1489 (95,9\%) completaron el seguimiento y aportaron la información solicitada para el análisis de costos. No se registraron muertes.

En el cuadro 1 se muestran las principales características clínicas y demográficas de la población que aportó datos al estudio de costos, agrupadas por categorías clínicas de ENI o NAC-Rx. Ninguno de los participantes tenía antecedentes de haber recibido vacunas antineumocócicas.

CUADRO 1. Características clínicas y demográficas de los casos que participaron en el estudio de costos, Región Metropolitana de Chile

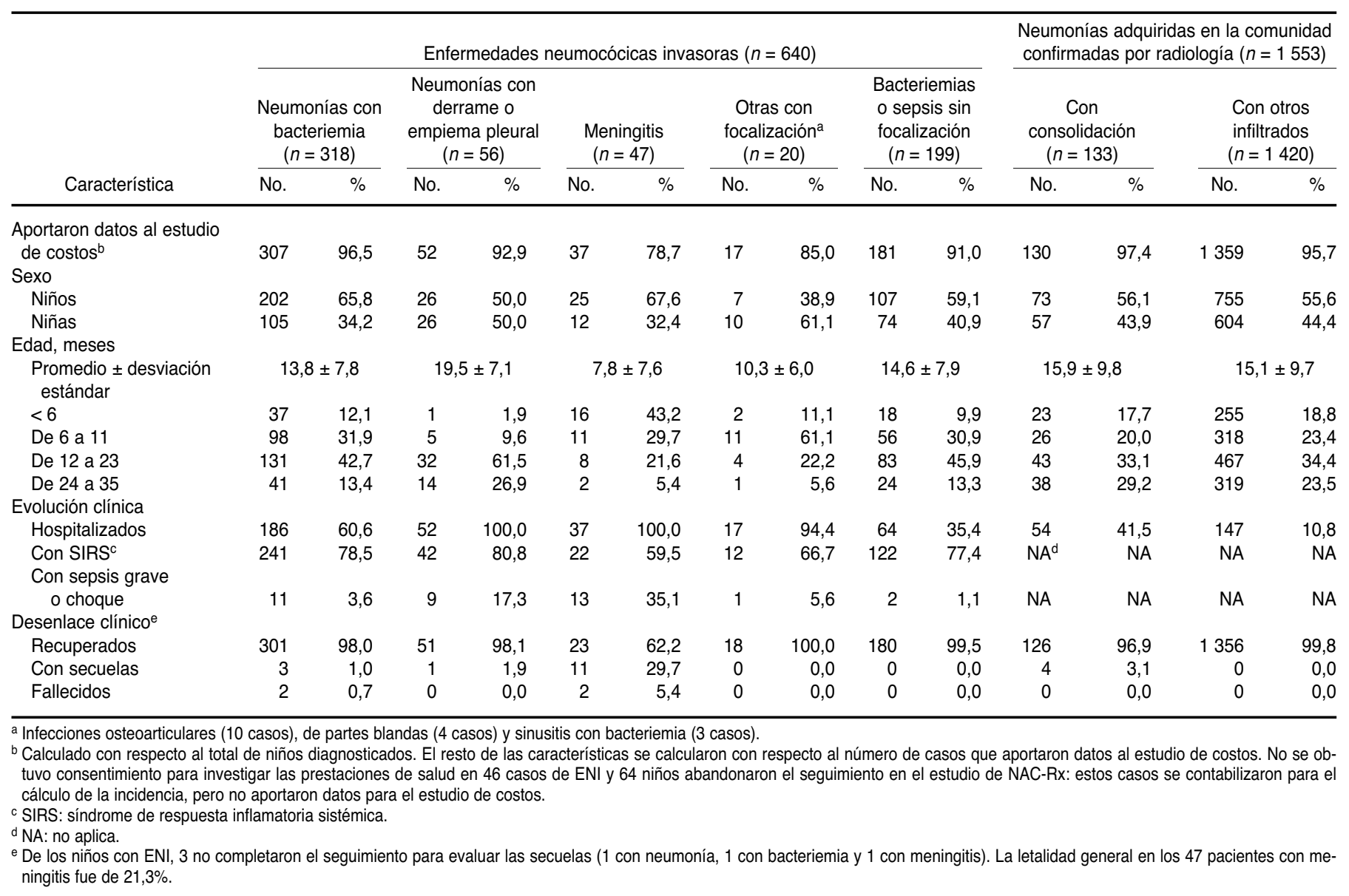


Estimación del número de casos de ENI sin documentación bacteriológica

Durante los 36 meses del estudio de costos de ENI, las unidades de urgencia de los hospitales estatales de la RM atendieron 864218 niños menores de 3 años que acudieron por causas no quirúrgicas; $25122(2,9 \%)$ pacientes se presentaron con temperatura rectal de $40{ }^{\circ} \mathrm{C}$ o más y se refirieron a su domicilio después de la evaluación médica. En 21707 (86,4\%) de ellos se obtuvieron muestras de sangre para hemocultivo, conforme a la práctica habitual para el cuidado de niños febriles en las unidades de urgencia, y en 234 $(1,1 \%)$ de estos exámenes se identificó S. pneumoniae. En los otros 3415 niños no se indicó hemocultivo pese a que cumplían los criterios de edad, fiebre y decisión de atención ambulatoria. Si se toma en cuenta que en $1,1 \%$ de los niños estudiados mediante hemocultivo se encontró S. pneumoniae, se puede esperar que en 38 de los pacientes con ENI no estudiados también estuviera presente esta infección. Así mismo, se infirió que de los
106299 menores de 3 años que acudieron a las unidades de urgencia con temperatura entre $39{ }^{\circ} \mathrm{C}$ y $40{ }^{\circ} \mathrm{C}$, en $744(0,7 \%)$ se habría aislado $S$. pneumoniae si se hubiera realizado un hemocultivo (31).

En cuanto a los servicios de atención primaria de urgencia de la RM, se atendieron 1110276 niños menores de 3 años durante el período de estudio. Si se aplican los mismos ajustes, se puede estimar que se atendieron $32198(2,9 \%)$ niños con fiebre alta; $136564(12,3 \%)$ con fiebre moderada y $1700(0,2 \%)$ casos de ENI que no fueron documentados porque el hemocultivo no formaba parte de las prácticas asistenciales habituales de esos servicios.

\section{Costos directos por episodio de ENI y NAC-Rx}

En el cuadro 2 se ofrece en detalle el promedio de prestaciones de salud que recibieron los pacientes con las diferentes formas clínicas y modalidades de atención de ENI y su valoración de acuerdo con las tarifas de FONASA en 2006.
Los costos directos promedio de las diferentes categorías de ENI aumentaron con los días de hospitalización, de US\$ 67,7 para las bacteriemias sin foco atendidas en forma ambulatoria, a US $\$ 2$ 590,4 para los casos de meningitis hospitalizados. La mayor parte (entre $70 \%$ y 75\%) del costo total de los casos hospitalizados estaba relacionada con la estadía hospitalaria. Todos los casos de meningitis, pleuroneumonía y otras ENI con focalización requirieron cuidados hospitalarios (cuadro 1). Una proporción considerable de las bacteriemias sin foco $(64,6 \%)$ y de las neumonías con bacteriemia $(39,4 \%)$ se resolvieron con cuidados ambulatorios solamente (cuadro 1); los costos de los casos hospitalizados fueron 6,3 y 8,5 veces más altos, respectivamente, que los atendidos en forma ambulatoria (cuadro 2).

En cuanto a los casos de NAC-Rx, también la estadía hospitalaria tuvo el mayor impacto sobre el costo total por caso (cuadro 3). Los costos de la atención médica de los pacientes hospitalizados fueron entre 7,3 veces (casos de neumonía no consolidante) y 14,6 veces (casos de

CUADRO 2. Servicios de salud y costos promedio asociados con el cuidado médico de niños de 0 a 35 meses de edad con enfermedades neumocócicas invasoras diagnosticadas y tratadas en establecimientos asistenciales estatales de la Región Metropolitana de Chile

\begin{tabular}{|c|c|c|c|c|c|c|c|c|c|c|c|c|c|c|}
\hline \multirow[b]{3}{*}{ Procedimientos y servicios } & \multicolumn{4}{|c|}{$\begin{array}{l}\text { Bacteriemias o sepsis } \\
\text { sin focalización }\end{array}$} & \multicolumn{4}{|c|}{ Neumonías con bacteriemia } & \multirow{2}{*}{\multicolumn{2}{|c|}{$\begin{array}{c}\text { Neumonías con } \\
\text { derrame o } \\
\text { empiema } \\
\text { Hospitalizados } \\
(n=52)\end{array}$}} & \multirow{2}{*}{\multicolumn{2}{|c|}{$\begin{array}{c}\text { Meningitis } \\
\text { Hospitalizados } \\
(n=37) \\
\end{array}$}} & \multirow{2}{*}{\multicolumn{2}{|c|}{$\begin{array}{c}\text { Otras con } \\
\text { focalización } \\
\text { Hospitalizados } \\
\quad(n=17)\end{array}$}} \\
\hline & \multicolumn{2}{|c|}{$\begin{array}{c}\text { Hospitalizados } \\
\quad(n=64)\end{array}$} & \multicolumn{2}{|c|}{$\begin{array}{c}\text { Ambulatorios } \\
\quad(n=117)\end{array}$} & \multicolumn{2}{|c|}{$\begin{array}{l}\text { Hospitalizados } \\
\quad(n=186)\end{array}$} & \multicolumn{2}{|c|}{$\begin{array}{c}\text { Ambulatorios } \\
(n=121)\end{array}$} & & & & & & \\
\hline & No. & US\$ & No. & US\$ & No. & US\$ & No. & US\$ & No. & US\$ & No. & US\$ & No. & US\$ \\
\hline \multicolumn{15}{|l|}{ Estadía hospitalaria, días ${ }^{b}$} \\
\hline Pediatría General & 4,3 & 206,0 & $N A^{c}$ & NA & 6,8 & 322,4 & NA & NA & 11,5 & 546,4 & 12,3 & 585,3 & 8,1 & 386,4 \\
\hline Cuidados Intermedios & 0,3 & 29,9 & NA & NA & 0,6 & 54,5 & NA & NA & 2,8 & 264,7 & 0,6 & 62,0 & 0,0 & 0,0 \\
\hline Cuidados Intensivos & 0,4 & 74,2 & NA & NA & 0,9 & 181,9 & NA & NA & 2,9 & 566,9 & 6,6 & 1299,4 & 0,1 & 11,6 \\
\hline \multicolumn{15}{|l|}{ Atención profesional ambulatoria } \\
\hline Unidades de emergencias & 1,6 & 26,4 & 2,1 & 33,5 & 1,6 & 26,6 & 2,3 & 37,7 & 2,1 & 34,3 & 2,1 & 34,6 & 1,8 & 28,6 \\
\hline Centros de salud primaria & 0,4 & 2,2 & 0,4 & 2,4 & 0,6 & 3,3 & 0,6 & 3,5 & 1,4 & 8,7 & 0,9 & 5,7 & 0,5 & 3,2 \\
\hline Visitas a especialistas & 0,7 & 6,4 & 0,6 & 6,3 & 0,9 & 8,8 & 0,7 & 6,6 & 1,8 & 17,7 & 3,3 & 32,3 & 2,4 & 23,6 \\
\hline Visitas a quinesioterapia & 0,1 & 0,2 & 0,2 & 0,4 & 0,8 & 1,4 & 0,5 & 0,9 & 3,3 & 5,6 & 1,3 & 2,3 & 0,8 & 1,4 \\
\hline \multicolumn{15}{|l|}{ Estudios diagnósticos } \\
\hline \multicolumn{15}{|l|}{ y procedimientos invasivos ${ }^{d}$} \\
\hline Hematología & 2,5 & 6,2 & 1,0 & 2,4 & 3,5 & 8,9 & 1,5 & 3,7 & 6,3 & 17,1 & 9,1 & 25,1 & 3,3 & 8,8 \\
\hline Química clínica & 4,6 & 29,3 & 1,0 & 5,3 & 8,5 & 56,9 & 1,6 & 9,3 & 14,0 & 93,3 & 29,4 & 186,9 & 4,9 & 30,8 \\
\hline Microbiología & 2,5 & 11,3 & 1,1 & 5,1 & 2,9 & 13,6 & 1,2 & 5,2 & 3,4 & 25,0 & 4,3 & 28,2 & 2,9 & 16,9 \\
\hline Radiología simple & 1,6 & 17,5 & 0,8 & 9,0 & 3,2 & 34,5 & 1,4 & 15,3 & 9,6 & 103,3 & 2,8 & 30,3 & 2,8 & 29,6 \\
\hline Imágenes complejas ${ }^{e}$ & 0,3 & 9,7 & $<0,1$ & 0,6 & 0,3 & 11,5 & $<0,1$ & 0,2 & 1,3 & 36,2 & 4,0 & 183,9 & 0,2 & 12,0 \\
\hline Procedimientos invasivos & 0,0 & 0,0 & 0,0 & 0,0 & $<0,1$ & 1,5 & 0,0 & 0,0 & 1,1 & 238,6 & 0,2 & 71,7 & 0,4 & 93,2 \\
\hline \multicolumn{15}{|l|}{ Tratamiento antimicrobiano ${ }^{\dagger}$} \\
\hline Tratamientos prescritos & 1,5 & & 0,9 & & 1,8 & & 1,1 & & 2,3 & & 2,3 & & 2,2 & \\
\hline Días con tratamiento & 9,3 & 10,5 & 7,4 & 2,7 & 12,4 & 15,0 & 8,9 & 4,9 & 25,4 & 37,2 & 25,1 & 42,7 & 20,2 & 11,0 \\
\hline Costo total por caso & & 429,7 & & 67,7 & & 740,8 & & 87,4 & & 1995,0 & & 2590,4 & & 657,1 \\
\hline
\end{tabular}

${ }^{a}$ Costos según las tarifas del Fondo Nacional de Salud (FONASA) de 2006, expresados en dólares estadounidenses a la tasa de cambio promedio de ese año (US $\$ 1,00=\$ 530,3$ pesos chilenos).

${ }^{\mathrm{b}}$ En FONASA, los costos de los servicios brindados durante la estadía hospitalaria se incluyen en el costo día-cama.

c NA: no aplica.

d Exámenes y procedimientos realizados en forma ambulatoria o durante la estadía hospitalaria.

e Estudios por ultrasonido, tomografía axial computarizada y resonancia magnética.

f Solo se contabilizaron los antibióticos administrados por 3 días o más. 
CUADRO 3. Servicios de salud y costos promedioa asociados con el cuidado médico de niños de 1 a 35 meses de edad con neumonía adquirida en la comunidad con confirmación radiológica diagnosticadas y tratadas en establecimientos asistenciales estatales de la Región Metropolitana de Chile

\begin{tabular}{|c|c|c|c|c|c|c|c|c|}
\hline \multirow[b]{3}{*}{ Servicios y procedimientos } & \multicolumn{4}{|c|}{ Neumonías con consolidación } & \multicolumn{4}{|c|}{ Neumonías no consolidantes } \\
\hline & \multicolumn{2}{|c|}{$\begin{array}{l}\text { Hospitalizados } \\
\quad(n=54)\end{array}$} & \multicolumn{2}{|c|}{$\begin{array}{c}\text { Ambulatorios } \\
(n=76)\end{array}$} & \multicolumn{2}{|c|}{$\begin{array}{l}\text { Hospitalizados } \\
\quad(n=147)\end{array}$} & \multicolumn{2}{|c|}{$\begin{array}{l}\text { Ambulatorios } \\
(n=1212)\end{array}$} \\
\hline & No. & US \$ & No. & US \$ & No. & US \$ & No. & US \$ \\
\hline \multicolumn{9}{|l|}{ Estadía hospitalaria, días } \\
\hline Pediatría General & 10,2 & 483,9 & $N A^{c}$ & NA & 4,8 & 227,3 & NA & NA \\
\hline Cuidados Intermedios & 0,9 & 83,2 & NA & NA & 0,1 & 9,1 & NA & NA \\
\hline Cuidados Intensivos & 0,9 & 168,5 & NA & NA & 0,6 & 121,1 & NA & NA \\
\hline Centros de salud primaria & 2,8 & 16,8 & 2,8 & 16,8 & 3,0 & 17,9 & 3,0 & 18,0 \\
\hline Visitas a especialistas & 0,1 & 0,9 & 0,0 & 0,0 & $<0,1$ & 0,1 & $<0,1$ & 0,1 \\
\hline Visitas a quinesioterapia & 6,0 & 10,4 & 3,8 & 6,5 & 4,7 & 8,1 & 3,7 & 6,4 \\
\hline \multicolumn{9}{|c|}{ Estudios diagnósticos y procedimientos invasivos ${ }^{d}$} \\
\hline Hematología & 3,1 & 6,6 & 0,1 & 0,3 & 1,9 & 4,3 & 0,2 & 0,4 \\
\hline Química clínica & 6,1 & 33,3 & 0,1 & 0,5 & 3,5 & 16,3 & 0,1 & 0,5 \\
\hline Microbiología & 2,7 & 13,8 & 0,1 & 0,4 & 1,7 & 9,1 & 0,04 & 0,2 \\
\hline Tratamientos prescritos & 1,0 & & 1,1 & & 0,7 & & 0,9 & \\
\hline Días con tratamiento & 8,6 & 3,7 & 8,7 & 2,7 & 5,2 & 2,3 & 7,0 & 1,9 \\
\hline Costo total promedio por caso & & 906,0 & & 61,9 & & 480,4 & & 65,4 \\
\hline
\end{tabular}

neumonía consolidante) más altos que los de los niños tratados en forma ambulatoria. Por otra parte, la duración promedio de las hospitalizaciones por neumonía consolidante duplicó la de los casos de neumonía no consolidante $(12,0$ frente a 5,5 días, respectivamente), lo cual se reflejó de igual manera en el costo total de la atención de estos pacientes. En contraste, los costos de los casos de NAC-Rx atendidos en forma ambulatoria no mostraron diferencias según el patrón radiológico.

\section{Costos según FONASA y dos clínicas privadas}

Para los 356 casos de ENI que requirieron hospitalización, el costo de la atención según las tarifas de FONASA fue en promedio de US\$ 1 056,2 por caso, mientras que en las dos clínicas privadas los costos fueron 4,2 y 9,4 veces mayores (US\$ 4 484,1 y US\$ 9 967,5) (cuadro 4). De manera similar, el costo directo promedio de un caso de NAC-Rx con estadía hospitalaria de un día o más fue de US\$ 594,8 según las tarifas de FONASA frente a US\$ 2962,7 y US\$ 6 578,4 en las dos clínicas privadas. Los costos de las prestaciones entregadas a los pacientes con NAC-Rx o ENI que no requirieron hospitalización fueron sustancialmente más bajos en los tres regímenes tarifarios, sin embargo la relación entre los costos según FONASA y los de las clínicas privadas fue del mismo orden que la observada en los casos hospitalizados (5,0 y 11,1 veces superiores, respectivamente).

\section{Estimación de la carga económica anual para FONASA}

En promedio, durante los tres años del estudio de costos se detectaron 213,5 casos anuales de ENI en niños de 0 a 35 meses en la RM de Chile (cuadro 5). Se estimó que si se hubieran realizado hemocultivos a todos los niños menores de 3 años que acudieron con fiebre alta a las unidades de urgencia y los servicios de atención primaria de urgencia, el número promedio de casos anuales hubiera sido de 344,1. La cifra hubiera llegado a 799,5 casos si también se hubieran realizado hemocultivos a todos los niños que se presentaron en esos servicios con fiebre moderada. Si la incidencia de ENI fuera homogénea en toda la población atendida por FONASA en el país, el total de casos anuales en niños de 0 a 35 meses de edad sería de 2131,1; si además se considera que los niños de 36 a 59 meses con ENI requerirían los mismos cuidados y procedimientos que los niños menores de 3 años, el costo de atención de los niños de 0 a 59 meses con ENI en todo el país según las tarifas de FONASA sería de US\$ 789 045,0.

Según los resultados de la vigilancia de los casos de NAC-Rx en seis comunas urbanas de la RM, la incidencia fue de 21,3 por cada 10000 niños de 1 a 35 meses de edad atendidos por FONASA (26). Si la tasa de ataque se mantuviera similar en todos los niños beneficiarios del subsistema público, se estima que la carga nacional anual promedio sería de 104 147,5 casos de NAC-Rx, con un costo total de US\$ 7899 067,5 en los casos hospitalizados y US\$ 5924 221,8 en los atendidos en forma ambulatoria (cuadro 6).

\section{DISCUSIÓN}

El costo de los cuidados médicos es un componente indispensable en el análisis 
CUADRO 4. Costos promedio de la atención médica de niños de 0 a 35 meses con enfermedades neumocócicas invasoras (ENI) y de 1 a 35 meses con neumonía adquirida en la comunidad con confirmación radiológica (NAC-Rx), según las tarifas de FONASA ${ }^{b}$ y de dos clínicas privadas de la Región Metropolitana de Chile

\begin{tabular}{|c|c|c|c|c|c|c|}
\hline \multirow[b]{2}{*}{ Diagnóstico y tipo de atención } & \multicolumn{2}{|c|}{ FONASA } & \multicolumn{2}{|c|}{ Clínica 1} & \multicolumn{2}{|c|}{ Clínica 2} \\
\hline & Hospitalizados $^{c}$ & Ambulatorios $^{d}$ & Hospitalizados $^{\mathrm{e}}$ & Ambulatorios & Hospitalizados $^{\mathrm{e}}$ & Ambulatorios \\
\hline \multicolumn{7}{|l|}{ Bacteriemia o sepsis sin foco } \\
\hline Atención profesional & 35,1 & 42,7 & 344,6 & 152,7 & 799,9 & 281,0 \\
\hline Exámenes y procedimientos ${ }^{g}$ & 74,0 & 22,3 & 232,1 & 75,6 & 560,8 & 166,1 \\
\hline Tratamiento antimicrobiano ${ }^{\mathrm{h}}$ & 10,5 & 2,7 & 46,3 & 11,0 & 77,3 & 35,0 \\
\hline Total & 429,7 & 67,7 & 2000,0 & 239,3 & 4587,0 & 482,1 \\
\hline Atención profesional & 40,1 & 48,8 & 553,1 & 177,9 & 1278,0 & 326,8 \\
\hline Exámenes y procedimientos & 126,9 & 33,7 & 401,7 & 120,6 & 928,3 & 244,5 \\
\hline Tratamiento antimicrobiano & 15,0 & 4,9 & 60,8 & 17,6 & 95,4 & 43,1 \\
\hline Total & 740,8 & 87,4 & 3366,0 & 316,1 & 7605,4 & 614,4 \\
\hline \multicolumn{7}{|l|}{ Neumonía con derrame o empiema } \\
\hline Estadía hospitalaria & 1378,1 & NA & 5293,8 & NA & 11680,3 & NA \\
\hline \multicolumn{7}{|l|}{ Meningitis } \\
\hline Estadía hospitalaria & 1946,7 & NA & 6567,0 & NA & 13862,7 & NA \\
\hline Atención profesional & 74,9 & NA & 1281,0 & NA & 3151,6 & NA \\
\hline Exámenes y procedimientos & 526,1 & NA & 1344,2 & NA & 3283,3 & NA \\
\hline Tratamiento antimicrobiano & 42,7 & NA & 237,1 & NA & 258,1 & NA \\
\hline Total & 2590,4 & NA & 9429,3 & NA & 20555,7 & NA \\
\hline \multicolumn{7}{|l|}{ Otras ENI con focalización } \\
\hline Estadía hospitalaria & 398,0 & NA & 2047,2 & NA & 4827,3 & NA \\
\hline Atención profesional & 56,9 & NA & 564,1 & NA & 1332,5 & NA \\
\hline Exámenes y procedimientos & 191,3 & NA & 488,6 & NA & 1145,3 & NA \\
\hline Tratamiento antimicrobiano & 11,0 & NA & 54,8 & NA & 78,2 & NA \\
\hline Total & 657,2 & NA & 3154,7 & NA & 7383,3 & NA \\
\hline \multicolumn{7}{|l|}{ Todos los casos de ENI } \\
\hline Estadía hospitalaria & 770,3 & NA & 3029,1 & NA & 6714,6 & NA \\
\hline Tratamiento antimicrobiano & 3,7 & 2,7 & 7,9 & 6,4 & 27,3 & 29,9 \\
\hline Total & 906,0 & 61,9 & 4620,7 & 295,4 & 10374,2 & 503,3 \\
\hline \multicolumn{7}{|l|}{ Neumonías no consolidantes } \\
\hline Estadía hospitalaria & 357,5 & NA & 1527,0 & NA & 3454,5 & NA \\
\hline Atención profesional & 58,5 & 43,3 & 546,3 & 213,6 & 1139,1 & 380,0 \\
\hline Exámenes y procedimientos & 62,1 & 20,1 & 275,1 & 92,8 & 573,2 & 122,5 \\
\hline Tratamiento antimicrobiano & 2,3 & 1,9 & 5,1 & 4,3 & 17,3 & 22,1 \\
\hline Total & 480,4 & 65,3 & 2353,5 & 310,7 & 5184,1 & 524,6 \\
\hline \multicolumn{7}{|l|}{ Todos los casos de NAC-Rx } \\
\hline Estadía hospitalaria & 459,1 & NA & 1995,2 & NA & 4536,4 & NA \\
\hline Atención profesional & 56,6 & 43,1 & 636,0 & 212,8 & 1344,6 & 378,5 \\
\hline Exámenes y procedimientos & 76,4 & 20,1 & 325,6 & 92,6 & 677,4 & 122,3 \\
\hline Tratamiento antimicrobiano & 2,7 & 2,0 & 5,9 & 4,5 & 20,0 & 22,6 \\
\hline Total & 594,8 & 65,2 & 2962,7 & 309,9 & 6578,4 & 523,4 \\
\hline
\end{tabular}

${ }^{a}$ Costos expresados en dólares estadounidenses a la tasa de cambio promedio de ese año (US $\$ 1,00=\$ 530,3$ pesos chilenos).

${ }^{b}$ FONASA: Fondo Nacional de Salud.

c En FONASA, los costos de los servicios brindados durante la estadía hospitalaria se incluyen en el costo día-cama.

¿ Exámenes y procedimientos realizados en forma ambulatoria o durante la estadía hospitalaria.

e En las clínicas privadas, la atención profesional brindada durante la estadía hospitalaria se cobra independiente del día-cama y la atención profesional abarca la brindada antes, durante y después de la hospitalización.

f NA: no aplica.

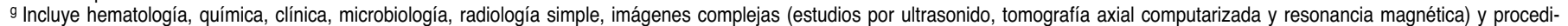
mientos invasivos.

${ }^{\mathrm{h}}$ Solo se contabilizaron los antibióticos administrados por 3 días o más. 
CUADRO 5. Carga económica estimada anual ${ }^{\mathrm{a}}$ secundaria al cuidado médico de los niños de 0 a 59 meses de edad con diferentes formas clínicas de enfermedad neumocócica invasora (ENI) en la Región Metropolitana de Chile

\begin{tabular}{|c|c|c|c|c|c|c|c|c|}
\hline \multirow[b]{2}{*}{ Grupo de edad y parámetro } & \multirow{2}{*}{$\frac{\text { Meningitis }}{\text { Hospitalizados }}$} & \multicolumn{2}{|c|}{$\begin{array}{c}\text { Neumonía } \\
\text { con bacteriemia }\end{array}$} & \multirow{2}{*}{$\begin{array}{c}\text { Neumonías } \\
\text { con derrame } \\
\text { o empiema } \\
\text { Hospitalizados }\end{array}$} & \multicolumn{2}{|c|}{$\begin{array}{c}\text { Bacteriemia } \\
\text { o sepsis sin foco }\end{array}$} & \multirow{2}{*}{$\begin{array}{c}\text { Otras } \\
\text { ENI con } \\
\text { focalización } \\
\text { Hospitalizados }\end{array}$} & \multirow[b]{2}{*}{ Total } \\
\hline & & Hospitalizados & Ambulatorios & & Hospitalizados & Ambulatorios & & \\
\hline \multicolumn{9}{|l|}{ En niños $<36$ meses } \\
\hline $\begin{array}{l}\text { Promedio anual de casos detectados } \\
\text { Promedio anual corregido de casos, } \\
\text { con hemocultivo en } 100 \% \text { de los } \\
\text { niños que acuden con fiebre alta } \\
\text { a las unidades de emergencia } \\
\text { y los servicios de atención primaria } \\
\text { de urgencias }\end{array}$ & 15,7 & 65,7 & 40,3 & 18,7 & 24,7 & 41,7 & 6,7 & 213,5 \\
\hline $\begin{array}{l}\text { Promedio anual corregido de casos, } \\
\text { con hemocultivo en } 100 \% \text { de } \\
\text { los niños que acuden con fiebre } \\
\text { moderada a las unidades } \\
\text { de emergencia y los servicios }\end{array}$ & & & & & & & & \\
\hline $\begin{array}{l}\text { de atención primaria de urgencias } \\
\text { Incidencia anual corregida, }\end{array}$ & 18,5 & 137,5 & 323,4 & 18,7 & 72,1 & 222,6 & 6,7 & 799,5 \\
\hline por 100000 niños $^{b}$ & 9,8 & 72,5 & 170,5 & 9,9 & 38,0 & 117,3 & 3,5 & 421,5 \\
\hline Estimado nacional de casos ${ }^{b}$ & 49,3 & 366,5 & 862,1 & 49,8 & 192,2 & 593,4 & 17,9 & 2131,1 \\
\hline \multicolumn{9}{|l|}{$\begin{array}{l}\text { En niños de } 36 \text { a } 59 \text { meses } \\
\text { Promedio anual de casos detectados }\end{array}$} \\
\hline en la Región Metropolitana & 1,0 & 13,7 & 2,0 & 7,3 & 1,3 & 2,3 & 2,0 & 29,6 \\
\hline Incidencia anual por 100000 niños $^{c}$ & 0,8 & 10,9 & 1,6 & 5,8 & 1,03 & 1,8 & 1,6 & 23,5 \\
\hline Estimado nacional de casos $^{c}$ & 2,7 & 37,4 & 5,5 & 19,9 & 3,5 & 6,3 & 5,5 & 80,8 \\
\hline Costo promedio por caso & 2590,4 & 740,8 & 87,4 & 1995,0 & 429,7 & 67,7 & 657,1 & \\
\hline $\begin{array}{l}\text { En niños de } 0 \text { a } 59 \text { meses con ENI } \\
\text { Estimación de la carga económica }\end{array}$ & & & & & & & & \\
\hline anual nacional & 134813,0 & 299223,0 & 75788,0 & 139202,0 & 84100,0 & 40596,0 & 15323,0 & 789045,0 \\
\hline
\end{tabular}

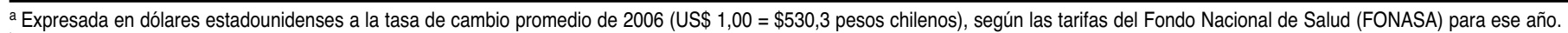

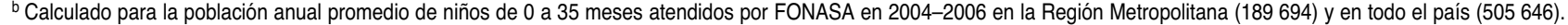

${ }^{c}$ Calculado para la población anual promedio de niños de 36 a 59 meses atendidos por FONASA en 2004-2006 en la Región Metropolitana (126 057 ) y en todo el país (344 131).

CUADRO 6. Carga económica estimada anuala secundaria al cuidado médico en niños de 1 a 35 meses con neumonía adquirida en la comunidad confirmada con radiología (NAC-Rx) en la Región Metropolitana de Chile

\begin{tabular}{|c|c|c|c|c|c|}
\hline \multirow[b]{2}{*}{ Parámetro } & \multicolumn{2}{|c|}{ Neumonías consolidantes } & \multicolumn{2}{|c|}{ Neumonías no consolidantes } & \multirow[b]{2}{*}{ Total } \\
\hline & Hospitalizados & Ambulatorios & Hospitalizados & Ambulatorios & \\
\hline \multicolumn{6}{|l|}{ Promedio anual de casos captados en la vigilancia } \\
\hline & 27,0 & 39,5 & 73,5 & 636,5 & 766,5 \\
\hline $\begin{array}{l}\text { Estimado anual de casos en las seis comunas vigiladas } \\
\text { Incidencia anual en niños de } 1 \text { a } 35 \text { meses, por } 100000\end{array}$ & 216,3 & 322,2 & 587,8 & 5180,6 & 6306,9 \\
\hline niños atendidos por FONASA, en las seis comunas vigiladas ${ }^{c}$ & 732,6 & 1091,4 & 1991,0 & 17547,7 & 21362,7 \\
\hline \multicolumn{6}{|l|}{ Estimado anual de casos en niños de 1 a 35 meses atendidos } \\
\hline por FONASA ${ }^{\mathrm{d}}$ en la Región Metropolitana & 1332,9 & 1985,5 & 3622,2 & 31924,8 & 38865,5 \\
\hline \multicolumn{6}{|l|}{ Estimado nacional de casos en niños de 1 a 35 meses } \\
\hline atendidos por FONASA ${ }^{e}$ en Chile & 3571,8 & 5320,6 & 9706,5 & 85548,6 & 104147,5 \\
\hline Costo promedio por caso de NAC-Rx & 906,0 & 61,9 & 480,4 & 65,4 & \\
\hline Carga económica anual para FONASA & 3236067,4 & 329343,4 & 4663000,1 & 5594878,4 & 13823289,3 \\
\hline
\end{tabular}

económico de cualquier intervención en salud, independientemente del tipo de análisis y de la perspectiva elegida para abordarlo (5-10). Como los costos pueden variar sustancialmente en función de las prácticas clínicas, el nivel de com- plejidad y la eficiencia de las empresas prestadoras y los gastos administrativos de los servicios de salud, los estudios económicos que utilizan datos ajenos a la comunidad destinataria de la intervención son de poca utilidad en los procesos de toma de decisiones locales (7-10). Más aún, las decisiones adoptadas a partir de estudios económicos cuyos parámetros no son consistentes con el escenario objeto de la intervención pueden afectar negativamente, en lugar de ele- 
var el impacto positivo de las inversiones en salud (10).

El presente estudio tiene varias fortalezas que reducen las posibilidades de sesgo en el muestreo y en la información recolectada. Primero, los investigadores se limitaron a pesquisar a los pacientes elegibles, a monitorear su evolución y a registrar los servicios de salud que recibían, pero no participaron directa o indirectamente en las indicaciones médicas previas o posteriores a la inclusión de los casos. Segundo, cada categoría diagnóstica investigada congregó un número considerable de casos. Y tercero, más de $90 \%$ de los casos elegibles de ENI y más de $95 \%$ de los casos elegibles de NAC-Rx completaron el seguimiento previsto. Esto avala que los paquetes de servicios descritos para los pacientes con ENI o NAC-Rx eran representativos de los procedimientos pediátricos estándar empleados en los establecimientos dependientes del Ministerio de Salud de Chile. Se debe destacar, además, que este estudio se insertó en sendos protocolos de vigilancia destinados a cuantificar la carga de morbilidad por ENI y NAC-Rx en niños atendidos por el sistema de salud estatal. Ponderados por las estimaciones de incidencia derivadas de estos estudios, los costos del cuidado médico por episodio calculados aquí muestran la carga económica que imponen estas enfermedades al presupuesto nacional administrado por FONASA.

Durante los tres años de estudio, en los establecimientos asistenciales estatales de la RM se diagnosticaron en promedio 243 casos anuales de ENI en niños menores de 5 años. Cerca de $65 \%$ de ellos requirió hospitalización y otras prestaciones de salud relativamente costosas, mientras los restantes eran casos de bacteriemia sin foco o neumonías neumocócicas no complicadas que se resolvieron satisfactoriamente con cuidados ambulatorios de bajo costo para FONASA.

En estudios epidemiológicos anteriores se demostró que la incidencia de ENI en la RM podría duplicarse si se extendiera la práctica de realizar hemocultivos a los niños que se presentan con fiebre alta en los servicios de urgencias del nivel primario (30) y se cuadruplicarían si se extendiera además a los niños menores de 3 años con síndrome febril moderado (31). Si bien las prácticas de atención pediátrica empleadas en la RM solo permiten detectar una pequeña parte de los casos pediátricos de ENI, en el pre- sente estudio se demostró que el costo económico adicional en que se incurriría por la atención de los casos no detectados actualmente es marginal, ya que la gran mayoría de ellos serían casos de ENI de curso clínico benigno - bacteriemia sin foco y neumonía no complicada- que requieren cuidados médicos ambulatorios de bajo costo. Dicho de otra forma, las cifras de incidencia de ENI en la RM contemplan la gran mayoría de los casos más graves y da cuenta de la mayor parte de la carga económica que imponen estas enfermedades sobre el presupuesto de FONASA.

A diferencia de los cultivos de sangre y otros fluidos normalmente estériles, cuya indicación se circunscribe al ámbito de la atención intrahospitalaria y a un grupo bien acotado de pacientes ambulatorios que acuden a las unidades de urgencia, la radiografía de tórax es un recurso diagnóstico asequible en todos los niveles de la red asistencial, ampliamente utilizado en la evaluación médica habitual de los niños chilenos con enfermedades respiratorias. La vigilancia de los casos de NAC-Rx en las seis comunas estudiadas de la RM mostró que uno de cada tres niños de 1 a 35 meses de edad atendidos por FONASA se sometió a un estudio radiológico de tórax durante el curso de una infección respiratoria aguda o de un síndrome febril; en 32,5\% de estos exámenes no se encontraron signos de neumonía y solo $13,6 \%$ de ellos confirmaron la necesidad de cuidados hospitalarios (26).

Como el uso de la radiografía no estuvo restringido por disponibilidad $\mathrm{ni}$ gravedad, se puede asegurar que la definición clínico-radiológica utilizada en este estudio fue adecuadamente sensible para capturar la incidencia real de NAC en la población vigilada $\mathrm{y}$, por ende, para apreciar la carga económica secundaria al cuidado médico de los niños con estas enfermedades. Lamentablemente, no hay estudios nacionales que permitan cotejar estas estimaciones de incidencia de NAC según su tipo radiológico y modalidad de tratamiento. En términos generales, sin embargo, la carga por hospitalizaciones proyectada a partir de la incidencia detectada en las seis comunas de la RM estudiadas es coherente con las estadísticas de egresos hospitalarios publicadas por el Ministerio de Salud (32).

En Chile no se han publicado estudios que analicen sistemáticamente los costos de las prestaciones de salud en estableci- mientos asistenciales del subsistema público. Ante la falta de información que represente adecuadamente la perspectiva de estos proveedores, en este estudio se valoraron los costos de los servicios médicos según las tarifas de pago fijadas por FONASA y los precios que cobrarían por esos mismos servicios dos clínicas privadas. Se debe recalcar que ninguno de estos tres regímenes tarifarios revela los costos reales de las prestaciones que recibieron los pacientes participantes. Se sabe que las tarifas de FONASA subvaloran los desembolsos en que deben incurrir tanto los establecimientos públicos como el Estado en general, ya que no toman en cuenta los gastos fijos de operación y administración y la depreciación de la infraestructura, entre otros componentes (33). Por el contrario, las tarifas de las clínicas privadas abarcan la totalidad de estos gastos, además de un margen de utilidades inherente a este tipo de empresas, pero no a los establecimientos estatales. En consecuencia, tiene sentido deducir que los costos reales del cuidado médico de las enfermedades examinadas en este estudio se encontrarían entre los calculados para FONASA y la clínica privada con tarifas más bajas.

Varios estudios anteriores encontraron una relación inversa entre la gravedad y los costos de la atención de las diferentes enfermedades causadas por S. pneumoniae (34-36). Mientras a las infecciones invasoras y las neumonías corresponden la gran mayoría de las muertes y casos graves, a las manifestaciones más benignas (como la otitis media y otras infecciones de las vías aéreas superiores) corresponde la mayor parte de la carga económica para los prestadores de salud y la sociedad en general, debido a su altísima incidencia. Esta relación también se observa en el presente estudio, tanto en los costos directos anuales de los casos de ENI con respecto a los de NAC-Rx, como en los costos de los casos de NAC-Rx que requieren hospitalización con respecto a los atendidos en forma ambulatoria.

Entre las limitaciones de este trabajo hay que señalar que no se analizaron los costos médicos de otras afecciones atribuibles a $S$. pneumoniae, como la otitis neumocócica y sus complicaciones, la neumonía en niños mayores de 35 meses y los casos de ENI y neumonías prevenibles por mecanismos de protección indirecta $(37,38)$. El diseño de este estudio tampoco contempló las repercusiones 
económicas de estas enfermedades sobre la sociedad en general.

En resumen, se cuantificaron los costos directos de la atención médica de enfermedades neumocócicas invasoras y neumonías, dos importantes problemas de salud infantil susceptibles de ser combatidos con vacunas antineumocócicas conjugadas. Si se toma en cuenta el tiempo y el esfuerzo invertido en recolectar estos datos, parece impracticable sugerir que la decisión de introducir una vacuna conjugada antineumocócica en los programas nacionales de inmunización deba apoyarse en estudios económicos que incor- poren este tipo de información local. Se recomienda priorizar la puesta en marcha de mecanismos sistemáticos de vigilancia que cumplan dos requisitos básicos: tener la sensibilidad suficiente para cuantificar, al menos, la mortalidad y la morbilidad grave atribuible a $S$. pneumoniae y mantener una estabilidad adecuada en el tiempo para poder monitorear el impacto de la vacuna después de su implementación. En lo que respecta a este estudio, la alta demanda asistencial y económica por NAC-Rx en niños de 0 a 3 años es una razón de salud pública tremendamente poderosa que apoya la apli- cación sistemática de vacunas antineumocócicas a niños chilenos.

Declaración de conflictos de intereses. Estos estudios se financiaron mediante un contrato de investigación entre el CVD-Chile y GlaxoSmithKline Biologicals. Rosanna Lagos, Alma Muñoz y Aníbal Espinoza recibieron honorarios de ese contrato en calidad de investigadores independientes. Rómulo Colindres y Ricardo Ruttiman son empleados de GlaxoSmithKline Biologicals. Ángela Dowes y Myron Levine no declaran conflictos de intereses.

\section{REFERENCIAS}

1. World Health Organization. Rotavirus vaccines. WHO position paper. Wkly Epidemiol Rec. 2007;82(32):285-95.

2. World Health Organization. Varicella vaccines. WHO position paper. Wkly Epidemiol Rec. 1998;73(32):241-8.

3. World Health Organization. Hepatitis A vaccines. WHO position paper. Wkly Epidemiol Rec. 2000;75(5):38-44.

4. World Health Organization. Pneumococcal conjugate vaccine for childhood immunization. WHO position paper. Wkly Epidemiol Rec. 2007;82(12):93-104.

5. Musgrove P, Fox-Rushby J. Cost-effectiveness analysis for priority setting. En: Jamison DT, Breman JG, Measham AR, Alleyne G, Cleason M, Evans D, et al., eds. Disease control priorities in developing countries. New York: Oxford University Press; 2006. Pp. 271-85. Hallado en http://www.ncbi.nlm.nih.gov/ books/bookres.fcgi/dcp2/ch15.pdf. Acceso el 12 de mayo de 2009.

6. World Health Organization. Economics of immunization: a guide to the literature and other resources. Geneva: WHO; 2004. (WHO/V\&B/ 04.02.) Hallado en www.who.int/vaccinesdocuments/DocsPDF04/www769.pdf. Acceso el 12 mayo de 2009.

7. Hutubessy R, Chisholm D, Tan-Torres T, WHO-CHOICE. Generalized cost-effectiveness analysis for national-level priority-setting in the health sector. Cost Eff Resour Alloc. 2003;8:1-8. Hallado en http://www.resource-allocation. com $/$ content $/ 1 / 1 / 8$. Acceso el 12 de mayo de 2009.

8. Schwartz B, Ortega-Sánchez I. Economic analyses of vaccines and vaccination programs. En: Levine MM, Kaper JB, Rappuoli R, Liu MA, Good M, eds. New generation vaccines. New York, Basel: Marcel Dekker; 2004. Pp. 107-15.

9. Hutubessy RC, Bendib LM, Evans DR. Critical issues in the economic evaluation of interventions against communicable diseases. Acta Trop. 2001;78:191-206.

10. Iglesias $\mathrm{CP}$, Drummonds MF, Rovira J, NEVALAT Group. Health-care decision making processes in Latin America: problems and prospects for the use of economic evalua- tion. Int J Technol Assess Health Care. 2005; 21:1-14.

11. Black S, Shinefield H, Fireman B, Lewis E, Ray $\mathrm{P}$, Hansen JR, et al. Efficacy, safety and immunogenicity of heptavalent pneumococcal conjugate vaccine in children. Pediatr Infect Dis J. 2000;19(3):187-95.

12. Klugman KP, Madhi SA, Huebner RE, Kohberger R, Mbelle N, Pierce N. A trial of a 9valent pneumococcal conjugate vaccine in children with and those without HIV infection. N Eng J Med. 2003;349(14):1341-8.

13. Cutts FT, Zaman SM, Enwere G, Jaffar S, Levine OS, Okoko JB, et al. Efficacy of ninevalent pneumococcal conjugate vaccine against pneumonia and invasive pneumococcal disease in The Gambia: randomized, doubleblind, placebo-controlled trial. Lancet. 2005; 365(9465):1139-46.

14. Hansen J, Black S, Shinefield H, Cherian T, Benson J, Fireman B, et al. Effectiveness of heptavalent pneumococcal conjugate vaccine in children younger than 5 years of age for prevention of pneumonia: updated analysis using World Health Organization standardized interpretation of chest radiographs. Pediatr Infect Dis J. 2006;25(9):779-81.

15. Madhi SA, Kuwanda L, Cutland C, Klugman $\mathrm{KP}$. The impact of a 9-valent pneumococcal conjugate vaccine on the public health burden of pneumonia in HIV-infected and -uninfected children. Clin Infect Dis. 2005;40(10):1511-8.

16. Eskola J, Kilpi T, Palmu A, Jokinen J, Haapakoski J, Herva E, et al. Efficacy of a pneumococcal conjugate vaccine against acute otitis media. N Engl J Med. 2001;344(6):403-9.

17. Prymula R, Peeters $\mathrm{P}$, Chroboc V, Kriz P, Novakova E, Kaliskova E, et al. Pneumococcal capsular polysaccharides conjugated to protein $\mathrm{D}$ for prevention of acute otitis media caused by both Streptococcus pneumoniae and non-typable Haemophilus influenzae: a randomised double-blind efficacy study. Lancet. 2006; 367(9512):740-8.

18. Sinha A, Levine O, Knoll MD, Muhib F, Lieu TA. Cost-effectiveness of pneumococcal conjugate vaccination in the prevention of child mortality: an international economic analysis. Lancet. 2007;369(9559):389-96.
19. Constenla DO. Economic impact of pneumococcal conjugate vaccination in Brazil, Chile and Uruguay. Rev Panam Salud Publica. 2008; 24(2):101-12.

20. Pan American Health Organization. Enhancing evidence-based capacity to make informed policy decisions on the introduction of new vaccines in the Americas: PAHO's ProVac Initiative. Washington, D.C.: PAHO; sin año Hallado en http://www.paho.org/English/ AD/FCH/IM/Provac Summary.htm. Acceso el 14 de julio de 2009.

21. República de Chile, Ministerio de Salud. Estadísticas de atenciones de salud. Santiago: MINSAL; 2009. Hallado en http:/ / deis.minsal. cl/index.asp. Acceso el 14 de julio de 2009.

22. Levine MM, Lagos R, Levine OS, Heitmann I, Enríquez N, Pinto ME, et al. Epidemiology of invasive pneumococcal infections in infants and young children in Metropolitan Santiago, Chile, a newly industrializing country. Pediatr Infect Dis J. 1998;17(4):287-93.

23. Lagos R, San Martín O, Erazo A, Avendaño A Levine MM. Epidemiología de las enfermedades invasoras causadas por Streptococcus pneumoniae en niños chilenos: proyecciones clínicas y de salud pública. Rev Chil Infectol. 2001;18(Supl 1):15-21.

24. Lagos R, Muñoz A, Valenzuela MT, Heitmann I, Levine MM. Population-based surveillance for hospitalized and ambulatory pediatric invasive pneumococcal disease in Santiago, Chile. Pediatr Infect Dis J. 2002; 21(12):1115-23.

25. Lagos R, Muñoz A, San Martín OS, Maldonado A, Hormazábal JC, Blackwelder WC, et al. Age- and serotype-specific pediatric invasive pneumococcal disease: insights from systematic surveillance in Santiago, Chile, 1994-2007. J Infect Dis. 2008;198(12):1809-17.

26. Lagos R, Muñoz A, Espinoza A, Moëne K, Hausdorff W, Ruttimann R, et al. Populationbased surveillance for suspected (S) and radiologically-confirmed ( $\mathrm{RxC}$ ) community acquired pneumonia (CAP) in children 1-35 months of age (MoA), in 6 municipalities (Mn) of the Metropolitan Region (MR), Chile. 5th International Symposium on Pneumococci and Pneumococcal Diseases; 2006 April 2-6; 
Alice Spring, Australia. Short Reports Book. Alice Spring, Australia: Tour Host Pty; 2006. Pp. 218. Hallado en http://www.tourhosts. com.au/archive/isppd5/pdf/abstracts book/FINAL_POSTER.pdf. Acceso el 12 de mayo de 2009.

27. World Health Organization, Vaccine Trial Investigator's Group. Standardization of interpretation of chest radiographs for the diagnosis of pneumonia in children. Geneva: WHO; 2001. (WHO/V\&B/01.35.) Hallado en http:// www.who.int/vaccine_research/documents/ en/pneumonia_children.pdf. Acceso el 12 de mayo de 2009

28. Central Nacional de Abastecimientos. Registro de compras, enero de 2005. Santiago: CENABAST. Hallado en http://www.cena bast.cl/registrocompras/compras2005.asp. Acceso el 19 julio 2009.

29. Banco Central de Chile. Tipo de cambio del dólar observado. Santiago: Banco Central de Chile; sin año. Hallado en http:/ / si2.bcentral. $\mathrm{cl} /$ basededatoseconomicos/951_455.asp? $\mathrm{f}=\mathrm{M}$ \&s=TC-OBS-MES. Acceso el 22 de julio de 2009.

30. Lagos R, Muñoz A, Espinoza M, Levine MM. Pneumococcal bacteremia among children $<36$ months of age (MoA) presenting with high fever to emergency care facilities of the primary and secondary level, in the Metropolitan Region (MR), Chile. 4th International
Symposium on Pneumococci and Pneumococcal Diseases; 2004 May 9-13; Helsinki, Finland. Abstract Book. Helsinki: ISPPD; 2004. Pp. 55. Hallado en http://www.congrex.fi/ isppd-4/images/abstracts.pdf. Acceso el 12 de mayo de 2009 .

31. Lagos RM, Muñoz AE, Levine MM. Prevalence of pneumococcal bacteremia among children $<36$ months of age presenting with moderate fever to pediatric emergency rooms of the Metropolitan Region (Santiago), Chile. Hum Vaccines. 2006;2(3):129-33.

32. República de Chile, Ministerio de Salud. Egresos hospitalarios 2004-2006. Santiago: MINSAL; 2007. Hallado en http://deis.minsal. $\mathrm{cl} /$ deis/codigo/neuw/hosp/indicadores hospital(2007)COMPLEJIDAD_20\%2006\% 2008\%20CORREGIDO2.htm. Acceso el 12 de mayo de 2009.

33. República de Chile, Ministerio de Salud. Verificación del costo esperado por beneficiario del conjunto priorizado de problemas de salud con garantías explícitas. Informe integrado. Enero de 2007. Santiago: MINSAL; 2007. Hallado en http://www.redsalud.gov.cl/ archivos/INFORMEFINAL_AGOSTO2007 versionoctubre07.pdf. Acceso el 12 de mayo de 2009.

34. Lieu TA, Ray GT, Black SB, Butler JC, Klein JO, Breinman RF, et al. Projected cost- effectiveness of pneumococcal conjugate vaccination of healthy infants and young children. J Am Med Assoc. 2000;283(11):1460-8.

35. Black S, Lieu TA, Ray GT, Capra A, Shinefield HR. Assessing costs and cost effectiveness of pneumococcal disease and vaccination within Kaiser Permanente. Vaccine. 2000;19(Suppl 1): S83-6.

36. Melegaro A, Edmunds WJ. Cost-effectiveness analysis of pneumococcal conjugate vaccination in England and Wales. Vaccine. 2004; 22(31-32):4203-14.

37. Ray GT, Whitney CG, Fireman BH, Ciuryla V, Black SB. Cost-effectiveness of pneumococcal conjugate vaccine: evidence from the first 5 years of use in the United States incorporating herd effects. Pediatric Infect Dis J. 2006;25(6) 494-501.

38. Grijalva CG, Nourti JP, Arbogast PG, Martin SW, Edwards KM, Griffin MR. Decline in pneumonia admissions after routine childhood immunization with pneumococcal conjugate vaccine in the USA: a time-series analysis. Lancet. 2007;369(9568):1179-86.

Manuscrito recibido el 23 de mayo de 2008. Aceptado para publicación, tras revisión, el 30 de diciembre de 2008.

ABSTRACT Objectives. To determine the direct medical costs of health care services for cases of invasive pneumococcal disease (IPD) and pneumonia acquired in the community and confirmed by radiology (NAC-Rx) among Chilean children.

Direct medical costs of invasive pneumococcal disease and radiologicallydiagnosed pneumonia among Chilean children

Methods. A prospective follow-up study of the health services delivered to 594 children 0-35 months of age with IPD and 1 489 children 1-35 months with NAC-Rx, diagnosed and treated by organizations within public health network of the Region Metropolitana de Chile. The value of the health services was established according to rates supplied by the Fondo Nacional de Salud (FONASA, the National Health Fund) and prices charged by two private clinics. The national IPD and NAC-Rx rates were estimated to calculate the total national economic burden for the population covered by state health insurance.

Results. The mean cost of cases requiring hospitalization was US\$ 1056.20 for IPD and US $\$ 594.80$ for NAC-Rx, while that of cases treated by out-patient services was US\$ 77.70 and US\$ 65.20, respectively. The cost of the same services for in-patient care at the private clinics was US\$ 4484.10 and US\$ 2962.70 at one clinic and US\$ 9967.50 and US\$ 6578.40 at the other. The estimated national annual cost of services for children under 5 years of age, according to FONASA rates, was US\$ 789045 for IPD and US\$ 13823289 for NAC-Rx.

Conclusions. The high demand for services and financial resources for NAC-Rx in children 0-3 years of age is a tremendously powerful public health reason to support the routine use of pneumococcal vaccination in Chilean children.

Key words Direct service costs; health care costs; pneumococcal infections; pneumococcal vaccines; child health; Chile. 